\title{
LAS METÁFORAS EMOCIONALES DE PROFESORES EN FORMACIÓN DE INFANTIL, PRIMARIA Y PSICOPEDAGOGÍA
}

\section{The emotional metaphors of Prospective Childhood, Primary, and Psychopedagogy Teachers}

\author{
Lucía Mellado*, María Rosa Luengo**, Juan Luis De la MONTAÑA*** \\ y María Luisa BERMEJO**** \\ * Dto. Economía de la Empresa y Contabilidad. Facultad de Ciencias Económicas y \\ Empresariales. UNED. Madrid. \\ * Dto. Didáctica Ciencias Sociales y de las Lenguas y Literaturas. Universidad de \\ Extremadura. España.
}

* Dto. Psicología y Antropología. Facultad de Educación. Universidad de Extremadura. España.

Recibido: 23-10-2015; Aceptado: 03-03-2016; Publicado: 30-05-2016

BIBLID [2386-3919 (2016) 34, 1; 179-195]

Ref. Bibl. LUCÍA MELLADO, MARÍA ROSA LUENGO, JUAN LUIS DE LA MONTAÑA y MARÍA LUISA BERMEJO. Las metáforas emocionales de profesores en formación de Infantil, Primaria y Psicopedagogía. Enseñanza \& Teaching, 34, 1-2016, 179-195.

RESUMEN: En este estudio se analizan las metáforas personales relacionadas con las emociones de estudiantes del grado de maestro durante el primer año de estudios y de estudiantes de Psicopedagogía durante su último año, procedentes de la Facultad de Educación de Badajoz. La muestra está formada por 35 estudiantes del Grado de Maestro de Educación Infantil, por 50 estudiantes del Grado de Maestro de Educación Primaria y por 46 estudiantes de la Licenciatura de Psicopedagogía, durante el curso 2010/2011, donde se les plantearon dos preguntas abiertas. En la investigación realizada encuadramos las metáforas en las cuatro categorías de Leavy, McSorley y Boté (2007): conductistas-transmisivas, cognitivas-constructivistas, situadas y autorreferenciadas. 
LUCÍA MELLADO, MARÍA ROSA LUENGO, JUAN LUIS DE LA MONTAÑA Y MARÍA LUISA BERMEJO LAS METÁFORAS EMOCIONALES DE PROFESORES EN FORMACIÓN DE INFANTIL, PRIMARIA Y PSICOPEDAGOGÍA

Los resultados indican que la mayoría de los participantes son capaces de conceptualizar sus roles en términos de metáforas. En los maestros en formación el mayor número de metáforas totales pertenecen a la categoría conductista-transmisiva, seguidas de la cognitiva/constructivista, autorreferenciada y situada. En cambio, los estudiantes de psicopedagogía expresan el mismo número de metáforas de la categoría conductista/transmisiva que de la cognitiva/constructivista, seguidas de las situadas y autorreferenciadas. En las metáforas emocionales el resultado es muy diferente: en los tres grupos el mayor número de metáforas emocionales pertenecen a la categoría cognitiva/constructivista, especialmente en los maestros, seguida de la autorreferenciada, expresando pocas metáforas emocionales de las categorías conductista-transmisiva y situada. Para los profesores en formación, la reflexión sobre sus propias metáforas, tanto las cognitivas como las emocionales, puede ser una poderosa herramienta para conceptualizar sus concepciones, actitudes, emociones y sus roles docentes y para iniciar cambios en los mismos.

Palabras clave: metáforas; emociones; formación del profesorado; educación infantil y primaria; psicopedagogía.

SUMMARY: This study analyzes the personal metaphors related to emotions of students in the first year of Maestro degree studies, and students of Psychopedagogy degree in the last year, from the Faculty of Education of Badajoz. The sample comes from 35 students of Maestro degree of Childhood Education, 50 students of Maestro degree of Primary Education, and 46 students of Psychopedagogy degree, during the year 2010/2011 where he raised two open questions. In research conducted metaphors in the four categories of Leavy, McSorley and Boté (2007): transmissive/ behavioral, cognitive/constructivist, situated and self-referential.

The results indicate that most of the prospective teachers were able to conceptualize their roles in the form of metaphors. The greatest number of metaphors expressed by the prospective primary and childhood teacher, belonged to the behaviourist/transmissive category, followed by the cognitivist/constructivist, selfreferential, and situative categories. However the psychopedagogy group, expressed the same number of metaphors in the categories behaviourist/transmissive and cognitivist/constructivist, followed by the situative and self-referential categories. In emotional metaphors the result is very different: The greatest number of emotional metaphors expressed by the three groups belonged to the cognitivist/constructivist category, especially in prospective primary and childhood teacher, followed by the self-referential category, with fewer in the behaviourist/transmissive and situative categories. For preservice teachers, reflecting on their own metaphors, both cognitive and emotional ones, can be a powerful tool to conceptualize their ideas, attitudes, emotions and teaching roles and to initiate changes in them.

Key words: metaphors; emotions; teacher training; nursery and primary education; psychopedagogy. 
LUCÍA MELLADO, MARÍA ROSA LUENGO, JUAN LUIS DE LA MONTAÑA Y MARÍA LUISA BERMEJO LAS METÁFORAS EMOCIONALES DE PROFESORES EN FORMACIÓN DE INFANTIL, PRIMARIA Y PSICOPEDAGOGÍA

\section{INTRODUCCIÓN}

Cuando el profesor en formación comienza su etapa universitaria, lo hace no sólo con un bagaje de conocimientos, sino con unos valores, creencias, roles, actitudes y emociones sobre la enseñanza, el aprendizaje y el rol del profesor, fruto de los muchos años de su propia escolaridad. Cada profesor elabora un pensamiento práctico profesional idiosincrásico, a partir de su experiencia personal y de la interacción social con el entorno que le rodea. A este pensamiento es difícil acceder y dotar de significados, ya que éste tiene unas percepciones sobre la enseñanza y el aprendizaje, que les resultan difíciles de articular y de expresar de una forma estructurada. El lenguaje que utiliza el docente para hablar de sus concepciones, roles y de su actividad profesional no suele ser literal y estructurado, sino más bien simbólico y de carácter metafórico.

Las metáforas utilizadas en el lenguaje del profesorado se han mostrado como un medio para articular el pensamiento y para establecer puentes entre las concepciones, el conocimiento práctico y la narrativa que describe la vida del aula (Ritchie, 1994). Estas metáforas personales tienen un gran componente afectivo y pueden ayudar a los profesores a tomar conciencia de sus sentimientos y emociones (Zembylas, 2004).

Desde la década de los 90 contamos con importantes estudios sobre las metáforas de los profesores (Bullough, 1992; Tobin et al., 1994). Sin embargo, coincidimos con Shaw et al. (2008) en que las metáforas deben seguir siendo objeto de investigación con el profesorado en aspectos menos tratados, como su relación con las emociones o su incidencia en el cambio de los modelos didácticos durante la formación inicial del profesorado.

En otros trabajos hemos analizado las metáforas de maestros en formación (Mellado et al., 2013) y de estudiantes de Psicopedagía (Mellado, 2014) en diferentes muestras. En este artículo analizamos las metáforas, y su componente emocional, de estudiantes de primer curso del Grado de Maestro de Infantil y Primaria, comparándolas con las de estudiantes del segundo ciclo de Psicopedagogía, al que la mayoría ha accedido después de realizar la carrera de Maestro. Con ello pretendemos determinar los cambios que se han producido en las metáforas de maestros en formación desde su entrada en la Universidad hasta cuatro años después al realizar el segundo ciclo.

\section{LAS METÁFORAS Y LOS PROFESORES EN FORMACIÓN}

Una metáfora es la sustitución o transposición de una idea o concepto por otro que tenga con él una cierta relación de semejanza objetiva o subjetiva para el emisor (Marcos, 1993). Las metáforas se han utilizado como un recurso expresivo en la literatura, en la oratoria, en la música y en numerosas expresiones artísticas y de comunicación. Desde la psicolingüística se estudia la correlación entre comportamiento lingüístico y procesos psicológicos subyacentes, por lo que la metáfora 
LUCÍA MELLADO, MARÍA ROSA LUENGO, JUAN LUIS DE LA MONTAÑA Y MARÍA LUISA BERMEJO LAS METÁFORAS EMOCIONALES DE PROFESORES EN FORMACIÓN DE INFANTIL, PRIMARIA Y PSICOPEDAGOGÍA

sería un mecanismo heurístico que se manifiesta lingüísticamente. Utilizando una metáfora de De Bustos (2000), las metáforas lingüísticas serían sólo la punta del iceberg de procesos cognitivos más básicos. En lingüística cognitiva, la metáfora es definida como un mecanismo cognitivo básico que consiste en la proyección de un dominio de conocimiento concreto, más conocido o estructurado, a otro dominio generalmente más abstracto y menos estructurado lingüísticamente (Alarcón y Díaz, 2014).

Las metáforas, por tanto, no son sólo un recurso expresivo, sino que tienen un significado más profundo ya que constituyen un mecanismo esencial de la mente, que estructura gran parte del sistema conceptual por medio de relaciones metafóricas (Martínez et al., 2001), y son un principio fundamental del pensamiento y la acción (Lakoff y Johnson, 1986).

Para Lakoff y Johnson (1986) lo esencial de la metáfora es comprender y experimentar una cosa en términos de otra. Las metáforas son como lentes, pantallas o filtros de lo que somos y de cómo nos vemos en determinada situación (Saban, 2010). Para Duffé (2004) las metáforas actúan como un código de valores compartidos por otros miembros de la comunidad cultural y lingüística y constituyen un medio de comprender el mundo. Expresado con la metáfora de Tobin y Tippins (1996), las metáforas son como semillas que germinan en nuevas ideas y conocimientos.

Las metáforas están presentes en numerosos aspectos de la investigación educativa. Hay metáforas para describir los paradigmas educativos, la formación del profesorado, el grupo clase, el aprendizaje, las estrategias de enseñanza o la evaluación. Las metáforas dan una visión holística de la vida del aula y tienden puentes entre la teoría y la narrativa que describe la vida del aula (Ben-Peretz et al., 2003; Buaraphan, 2011; Korthagen, 2010; Zhao, Coombs y Zhou, 2011). El estudio de las metáforas ha sido defendido como una potente herramienta que estimula y potencia la reflexión de los profesores (Gosselin y Meixner, 2015).

Un resultado importante de la investigación es que las metáforas personales de los profesores influyen poderosamente en su conducta docente en el aula (BouJaoude, 2000; Gurney, 1995). Numerosos trabajos han mostrado que los profesores realizan cambios en sus concepciones y en su práctica en el aula cuando son capaces de construir nuevos modelos en un proceso de reflexión crítica y al mismo tiempo construyen nuevas metáforas compatibles con tales cambios (Russell y Hrycenko, 2006; Thomas y Beauchamp, 2011; Tobin et al., 1994).

Los profesores construyen sus metáforas a partir de sus antecedentes escolares y de su propia experiencia personal (Ben-Peretz et al., 2003), por lo que tienen un fuerte componente afectivo (Tobin y Tippins, 1996). Las metáforas son un poderoso instrumento que sirve de puente para unir el mundo cognitivo y afectivo y que pueden ayudar a los profesores a tomar conciencia de sus sentimientos y emociones (Rebollo et al., 2013; Lakoff y Johnson, 1986). En la enseñanza hay aspectos emocionales, irracionales desde el punto de vista cognitivo, que influyen en las acciones y en la toma de decisiones de los profesores (Damasio, 2010). 
LUCÍA MELLADO, MARÍA ROSA LUENGO, JUAN LUIS DE LA MONTAÑA Y MARÍA LUISA BERMEJO LAS METÁFORAS EMOCIONALES DE PROFESORES EN FORMACIÓN DE INFANTIL, PRIMARIA Y PSICOPEDAGOGÍA

Las emociones son muy importantes para todos los profesores, pero especialmente para los profesores en formación. Las primeras experiencias de enseñanza durante el Practicum están llenas de tomas de decisiones, son emocionalmente muy fuertes y pueden ser traumáticas para los futuros profesores, que en esta etapa son especialmente vulnerables, generándoles emociones negativas como ansiedad, inseguridad, irritación o decepción. Estas emociones negativas pueden fijar estrategias conductistas, dirigidas hacia el control y la supervivencia, que son muy resistentes al cambio en el futuro.

Nuestro trabajo, en la línea de los realizados por Leavy, McSorley y Boté (2007) y López-Luengo et al. (2015), está orientado a determinar la evolución de las metáforas personales de futuros profesores durante sus estudios universitarios, pero además consideramos importante incluir el componente emocional y distinguir las metáforas emocionales.

\section{PROBlema DE INVESTIGACIÓN}

Los objetivos de este estudio son:

1. Averiguar las metáforas personales de una muestra de maestros en formación de los Grados de Educación Infantil y de Educación Primaria al principio de su formación inicial y de estudiantes del segundo ciclo de Psicopedagogía.

2. Clasificar las metáforas personales de la muestra dentro de las categorías: conductista-transmisiva, cognitiva-constructivista, situadas y autorreferenciadas.

3. Analizar las metáforas personales relacionadas con las emociones y comparar con las generales.

4. Determinar la evolución de las metáforas durante los estudios universitarios, comparando los estudiantes de primer curso del grado de Maestro con los estudiantes de último curso de Psicopedagogía.

\section{Metodología}

La investigación se ha realizado el curso 2010-2011 en la Facultad de Educación de la Universidad de Extremadura. La muestra la componen estudiantes del Grado de Maestro de Infantil y Primaria en el primer año de formación, durante los primeros contactos con la carrera, y estudiantes del último curso de Psicopedagogía. La muestra se ha elegido por facilidad de acceso y aunque no pretende ser representativa estadísticamente, se han seleccionado los grupos que por edad y formación son representativos de los estudiantes de estas carreras.

La distribución de la muestra de educación primaria es de 19 hombres y 31 mujeres, lo que representa un $40,0 \%$ de hombres y un $60,0 \%$ de mujeres. En cuanto a la edad, la mayoría tiene menos de 20 años porque son alumnos que estudian 
LUCÍA MELLADO, MARÍA ROSA LUENGO, JUAN LUIS DE LA MONTAÑA Y MARÍA LUISA BERMEJO LAS METÁFORAS EMOCIONALES DE PROFESORES EN FORMACIÓN DE INFANTIL, PRIMARIA Y PSICOPEDAGOGÍA

por primera vez una carrera universitaria. La muestra de educación infantil es de 31 mujeres y 4 hombres, lo que representa un $88,6 \%$ y un $11,4 \%$, respectivamente. La mayoría también tiene menos de 20 años. La muestra de Psicopedagogía está formada por 8 hombres y 38 mujeres, lo que representa un 17,4\% y un 82,6\% respectivamente. Un $71,3 \%$ de los estudiantes de Psicopedagogía han realizado anteriormente la Diplomatura de Maestro, lo cual nos permite hacer un seguimiento de la evolución de las metáforas en cuatro años, desde que entraron en la Universidad hasta después de haber realizado los distintos periodos del Practicum en las diplomaturas y en la propia licenciatura de Psicopedagogía.

El predominio de mujeres en la muestra refleja la feminización de la profesión de Maestro y quizás también la relación entre la elección de la carrera y los estereotipos de género asociados a la profesión (Gutiérrez y Luengo, 2003).

El procedimiento de recogida de datos ha sido un cuestionario anónimo en el que además del sexo y la edad se hacen dos preguntas abiertas:

1. Cuando impartas clases en un aula, ¿con qué metáforas te identificarías?

2. Explica las razones que te llevan a identificarte con esas metáforas.

Antes de pasar el cuestionario se les informó sobre el significado y la importancia de las metáforas en educación, pero no se les dio ningún ejemplo de metáforas personales para no condicionar sus respuestas.

En nuestro estudio adaptamos las cuatro categorías de Leavy et al. (2007): la conductista-transmisiva: el alumno como un aprendiz pasivo, el profesor como un transmisor de información y la motivación dependiente de refuerzos externos, que tiene además la característica de ser una clase centrada en el profesor y el contenido; la cognitiva-constructivista: el alumno como un agente activo en el proceso de construcción de su propio conocimiento y el profesor como un facilitador del proceso; la situada o de aprendizaje social: el aprendizaje es situado en el contexto social y la motivación surge del compromiso con la comunidad educativa, estando con la cognitiva-constructivista centrada en el alumno y el aprendizaje; la autorreferenciada, que son metáforas de difícil clasificación y que tienen un componente egocéntrico, cuyo significado es necesario analizar en cada caso.

Estos modelos nos permiten utilizar las metáforas personales de los profesores para determinar las concepciones y los modelos implícitos de enseñanza asociados a las mismas. Sin embargo, los trabajos anteriores se han centrado más en los aspectos cognitivos que en los emocionales (Mellado et al., 2012). El objetivo de este artículo es analizar las metáforas emocionales de los profesores y determinar si su distribución en modelos presenta diferencias con las metáforas personales generales.

Hemos incluido también algunos proverbios, sintagmas convencionales o modismos expresados por los profesores. Los modismos son frases cortas y fijas con sentido privativo, cuyo significado conjunto está asumido por el colectivo cultural y lingüístico, pero que no se deduce literalmente de las palabras que lo 
LUCÍA MELLADO, MARÍA ROSA LUENGO, JUAN LUIS DE LA MONTAÑA Y MARÍA LUISA BERMEJO LAS METÁFORAS EMOCIONALES DE PROFESORES EN FORMACIÓN DE INFANTIL, PRIMARIA Y PSICOPEDAGOGÍA

forman. Para Gibbs, 1992 (citado por Duffé, 2004), los modismos son como «metáforas cristalizadas» que encierran significados muy arraigados en una comunidad.

Con respecto a las emociones en nuestro estudio clasificamos las emociones en primarias o sociales y en positivas, negativas o neutras. Todas las emociones analizadas han sido reconocidas como tales por varios autores (Bisquerra, 2000; Borrachero et al., 2014; Brígido et al., 2013; Damasio, 2010; Díaz y Flores, 2001; Fernández-Abascal, Martín y Domínguez, 2001; Goleman, 1996; Rebollo et al., 2008).

Se realizó un análisis descriptivo de frecuencias en cada grupo y categoría y se discutieron las razones por las que se identifican con las metáforas, contrastando los resultados con anteriores estudios.

\section{DisCusión De lOS RESUltados}

\subsection{Metáforas personales expresadas por los tres grupos}

El número total de metáforas descritas es de 57 en educación primaria, de 64 en educación infantil y de 55 en psicopedagogía. En el Gráfico 1 se representan los porcentajes de las metáforas expresadas por los tres grupos en las cuatro categorías de análisis.

Para encuadrar las metáforas en una categoría es fundamental analizar el significado que se les da ya que las metáforas son como espadas de doble filo (Ritchie et al., 2006) y para comprenderlas es necesario analizar el contexto y los significados asignados por el profesor. Sin embargo, en algunos casos los profesores no explican suficientemente el sentido, por lo que hemos asignado la metáfora a la categoría que en nuestra opinión mejor se encuadraba.

En los dos grupos de maestros el mayor número de metáforas se encuentra dentro de la categoría conductista/transmisiva, donde la metáfora dominante es la del profesor como transmisor de conocimientos, habilidades y destrezas y la del alumno la de receptor pasivo de estos conocimientos (Cassel y Vincent, 2011; Levy et al., 2007; Martínez et al., 2001; Seung et al., 2011). Si, como señalan numerosas investigaciones (Sillman y Dana, 2001; Tobin et al., 1994), los profesores en formación toman como referencia los modelos de enseñanza en los que ellos mismos fueron enseñados, el gran número de metáforas conductistas/transmisivas expresadas por los futuros maestros puede ser un reflejo del tipo de enseñanza que ellos mismos recibieron. También puede ser porque al no tener experiencia de enseñanza se encuentran en la etapa evolutiva que Fuller y Brown (1975) denominan de supervivencia, centrada en ellos mismos y no en la enseñanza y en los alumnos. 
LUCÍA MELLADO, MARÍA ROSA LUENGO, JUAN LUIS DE LA MONTAÑA Y MARÍA LUISA BERMEJO LAS METÁFORAS EMOCIONALES DE PROFESORES EN FORMACIÓN DE INFANTIL, PRIMARIA Y PSICOPEDAGOGÍA

GRÁFICO 1

Tipos de metáforas generales en los tres grupos

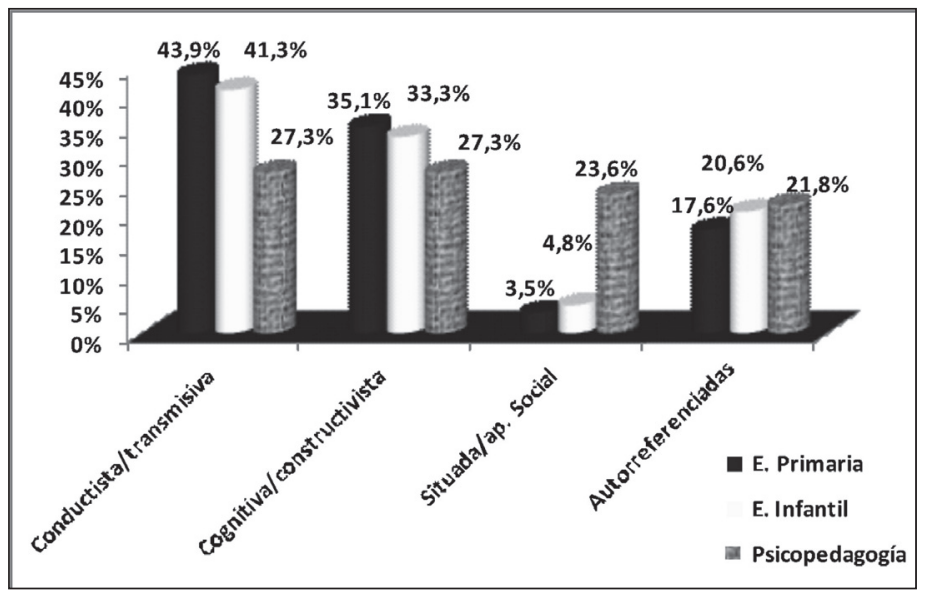

Un porcentaje significativo de metáforas de los dos grupos de maestros son cognitivas/constructivistas, donde el profesor se considera un educador y facilitador, y el alumno un aprendiz activo que construye poco a poco un aprendizaje significativo (Boujaoude, 2000). Otro resultado es el pequeño porcentaje de metáforas situadas en los maestros en formación. Son metáforas identificadas como iluminación, viaje, trayecto, descubrimiento, exploración, búsqueda, guía, etc. (Gurney, 1995; Leavy et al., 2007; Ritchie, 1994; Sillman y Dana, 2001). En algunos casos puede ser debido a que son más difíciles de expresar y por otro a que los estudiantes al iniciar sus estudios de maestro asumen un aprendizaje más individualizado, en detrimento de las metáforas que hacen referencia a una enseñanza social y colaborativa. Destacamos el porcentaje de las metáforas que están encuadradas en la categoría autorreferenciadas y que representan a animales (Lantolf y Bobrova, 2014). Estas expresiones permiten comunicar en pocas palabras contenidos de gran complejidad. Hay participantes que se identifican con animales, y al igual que al escuchar los cuentos en su niñez había un animal preferido antropomórfico, los futuros profesores se identifican con una o varias características de animales para desempeñar su rol en el aula.

Los resultados del grupo de psicopedagogía son muy diferentes de los dos grupos de maestro. Los estudiantes de psicopedagogía expresan el mismo número de metáforas conductistas/transmisivas que cognitivas/constructivistas (27,3\%), seguidas de las situadas $(23,6 \%)$ y autorreferenciadas $(21,8 \%)$. Destacamos el gran aumento de las situadas $(23,6 \%)$, respecto a las expresadas por los maestros, manteniéndose un porcentaje similar en las autorreferenciadas.

Sólo la metáfora del pentagrama, indicada por una estudiante de psicopedagogía con estudios previos de Maestra de Educación Musical, expresa una relación 
LUCÍA MELLADO, MARÍA ROSA LUENGO, JUAN LUIS DE LA MONTAÑA Y MARÍA LUISA BERMEJO LAS METÁFORAS EMOCIONALES DE PROFESORES EN FORMACIÓN DE INFANTIL, PRIMARIA Y PSICOPEDAGOGÍA

con un contenido curricular. El resto son expresiones de la visión general sobre la enseñanza, formada en los años de escolaridad, independientemente de los contenidos concretos (Mahlios et al., 2010; Mellado et al., 2012).

\subsection{Metáforas relacionadas con las emociones}

Exponemos en el Gráfico 2 los porcentajes de las metáforas emocionales de los tres grupos. El número total de metáforas emocionales en los grupos de maestros de educación primaria e infantil han sido 28 y 34 respectivamente, y de 24 en el grupo de psicopedagogía.

A diferencia de las metáforas globales, donde el mayor número pertenecían a la categoría conductista/transmisiva, en las emocionales hay un gran porcentaje de metáforas cognitivas/constructivistas, especialmente en los maestros, seguidas de las autorreferenciadas.

GRÁFICO 2

Tipos de metáforas emocionales en los tres grupos

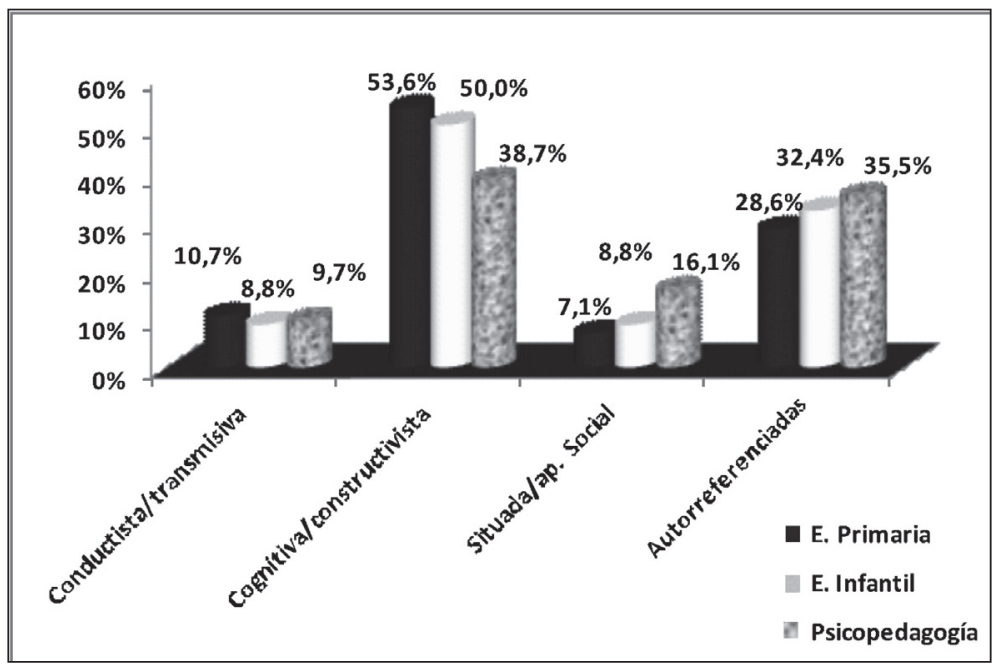

A continuación, mostraremos algunas de las metáforas emocionales más significativas de los tres grupos, según las razones que los participantes han dado para identificarse con estas metáforas.

\subsubsection{Metáforas que reflejan emociones primarias positivas}

Felicidad: Entre las metáforas que identificamos con la felicidad están las sensoriales como los días cálidos o la de despertar el placer de la creatividad. Otro se 
LUCÍA MELLADO, MARÍA ROSA LUENGO, JUAN LUIS DE LA MONTAÑA Y MARÍA LUISA BERMEJO LAS METÁFORAS EMOCIONALES DE PROFESORES EN FORMACIÓN DE INFANTIL, PRIMARIA Y PSICOPEDAGOGÍA

identifica con un pintor creativo que plasma sus ideas buscando la parte más original. Estas dos últimas metáforas reflejan emociones estéticas según Goleman (1996) y Bisquerra (2000), donde identifican su futuro rol de profesores con el placer de la creación. A una participante le gustaría ser la sonrisa de los alumnos representando la alegría. Otra persona hace alusión al refrán de «no es más feliz quien más tiene, sino el que menos necesita». Hay varias metáforas que indican felicidad y que se identifican con animales, como: felices como una lombriz, o una perdiz, o el vuelo de una mariposa (Bullough, 1992), o pez en el agua, esta última expresada por cuatro estudiantes de psicopedagogía. La metáfora del pez en el agua también ha sido identificada por López-Luengo et al. (2015) en profesores de educación infantil. En otra metáfora se alude a la felicidad con el significado de unas castañuelas.

\subsubsection{Metáforas que reflejan emociones positivas sociales}

Diversión: Estas se diferencian de las que hemos encuadrado en la felicidad, en que tienen un significado más social. Una persona se identifica con una metáfora visual y olfativa como es la flor, aunque esta metáfora suele asignarse por los profesores en formación más al desarrollo de los niños que a sí mismos (Saban, 2010). También se expresa la sensación de placer en el refrán, "sarna con gusto no pica». Dos futuros maestros de primaria y dos de infantil se ven como payasos, graciosos y divertidos. En este apartado es donde más abundan las metáforas de los futuros maestros de infantil. La metáfora de la primavera es señalada con dos significados: una se ve a sí misma como la primavera, enérgica, joven y con entusiasmo y otro asigna la metáfora a la clase, alegre y llena de colores. El mismo significado le asigna a la clase un futuro profesor de primaria, al verla como una casa llena de soles. Otras metáforas del grupo de infantil son las referidas a los niños como pequeños soles o como la música. Entre las personales los del grupo de infantil se ven como juguetes, porque lo más importante en la vida de los niños es el juego, o como un personaje fantástico y teatral (Seung et al., 2011) o alegre como un solete.

Seguridad: En los futuros maestros hay varias metáforas familiares de hermano mayor que expresan seguridad, y en el de psicopedagogía la del amigo. Otro futuro maestro de infantil se identifica con un canguro, que protege a los niños en su bolsa.

Adaptación/flexibilidad: Las metáforas del profesor de goma o del junco flexible, que no se rompería ante las adversidades y se amoldaría a los alumnos y a sus necesidades, expresan perfectamente la necesidad de adaptación. El mismo significado tiene la metáfora del camaleón, frecuentemente identificada en otros estudios (Boujaoude, 2000; Buaraphan, 2011; Tobin et al., 1994). Una metáfora que en la explicación indica este significado es la del arquitecto, para adaptarse a las mentes de los niños, con un sentido semejante a la encontrada en otros estudios de profesores en formación (Saban, 2010) para referirse al rol social de contribuir a formar la mente de cada uno de los estudiantes. 
LUCÍA MELLADO, MARÍA ROSA LUENGO, JUAN LUIS DE LA MONTAÑA Y MARÍA LUISA BERMEJO LAS METÁFORAS EMOCIONALES DE PROFESORES EN FORMACIÓN DE INFANTIL, PRIMARIA Y PSICOPEDAGOGÍA

Perseverancia/paciencia: Un futuro maestro de primaria se identifica con una tortuga (Bullough, 2010), que tiene paciencia, camina lentamente y además es pacífica. Otra metáfora es la del joyero tallando diamantes, que relaciona con la perseverancia, pero que tiene un significado muy conductista al considerar al estudiante como materia prima que el profesor tiene que pulir y moldear (Buaraphan, 2011; López-Luengo et al., 2015).

Confianza: Uno de los estudiantes no indica una metáfora propiamente dicha, sino que resalta que quiere ser el profesor en el que confían los niños. Con este significado otro se identifica con el maestro amigo. En el grupo de infantil se expresa la metáfora del gato, que es cariñoso y de confianza, pero que a su vez se hace respetar.

Orgullo: Se sienten orgullosos y respetados de la profesión, como el león, pero significando que también quieren ser cercanos a los niños. La metáfora del rey león ha sido utilizada por profesores en formación en otros estudios con el significado de cuidar y proteger a los niños (Seung et al., 2011). Otra futura profesora de psicopedagogía comenta que le gustaría ser un águila, animal de altos vuelos; se identifica con el espíritu de la valentía, del orgullo, para intentar alcanzar las metas propuestas.

Comprensión: En la actitud tolerante y respetuosa hacia los sentimientos o actos de otra persona se expone la metáfora de un animal como el perro labrador, que es fiel y comprensivo.

Empatía: En este apartado, según Bisquerra (2000) y Goleman (1996), incluimos dos metáforas del grupo de psicopedagogía, que se ven como modelos para los estudiantes.

Amor/cariño: Entre las metáforas que expresan amor y afecto destacamos la de la madre o la maternal, señaladas en los tres grupos (Thomas y Beauchamp, 2011; Tobin et al., 1994), la maestra cercana a los niños, con el significado de darles afecto, y la de la manta que representa el calor y el cariño, esta última, similar al edredón recogida por Sillmam y Dana (2001). Un futuro maestro de primaria se identifica con un oso de peluche, y uno de infantil asigna a los niños la metáfora del peluche, significando en ambos casos cariño y calidez. En infantil una maestra en formación señala que quiere ser como un primer beso de amor, para estar siempre en el recuerdo de los niños. Otra metáfora de cariño es querer ser el corazón de los niños.

Generosidad: A pesar de que el topo indica una connotación negativa ya que en algunos casos indicaría estrechez de miras por la ceguera, una alumna de Infantil expresa todo lo contrario ya que le gustaría ser un «topo para acercarme a cada uno de mis alumnos, ciega y sin prejuicios». También en muchas ocasiones se usa el símil entre profesores y médicos, y se recurre a la metáfora de este futuro profesor "Como el médico, que cuida y salva a los niños». En ambos casos, estas 
LUCÍA MELLADO, MARÍA ROSA LUENGO, JUAN LUIS DE LA MONTAÑA Y MARÍA LUISA BERMEJO LAS METÁFORAS EMOCIONALES DE PROFESORES EN FORMACIÓN DE INFANTIL, PRIMARIA Y PSICOPEDAGOGÍA

metáforas manifiestan la necesidad de atender a cada uno de los alumnos según sus necesidades y sus posibilidades.

Motivación: Varios profesores de los tres grupos se identifican con la luz que alumbra el camino, metáforas de guías identificadas en otros estudios (Gurney, 1995; Thomas y Beauchamp, 2011; Tobin et al., 1994). Con el mismo significado de guía para los alumnos es la metáfora del sol para inspirarlos y acompañarlos en el camino (Buaraphan, 2011). Otra metáfora con este significado es la del agua motivadora/alentadora, esta última muy común en profesores con la orientación cognitiva/constructivista (Treagust y Harrison, 2000).

Entusiasmo: Estas metáforas son parecidas a las anteriores, pero se hace más hincapié en la figura del profesor que en su proyección hacia el estudiante. Un futuro maestro de primaria se considera un chorro de agua, por el entusiasmo en su labor. Un futuro maestro de infantil se ve como un cuento del que los niños aprenden cosas de la vida; en esta metáfora el profesor tiene que tener sensibilidad, imaginación y creatividad y ser un contador de historias, para motivar a los alumnos. Uno de primaria se ve como una ventana abierta y llena de posibilidades. Lorsbach et al. (1992) señalan que, cuando una profesora cambió la metáfora del juez justo por la de una ventana en la mente del estudiante, cambió a mejor el clima del aula y la forma de evaluar.

Placer/disfrute: Varios participantes se asignan metáforas de animales, relacionadas con el placer: libre como un pájaro, o como una mariposa, o como una paloma, para llegar a todos y enseñarles que es posible conseguir cualquier cosa. Otra se ve soñadora como un delfín, porque realizará lo que siempre ha querido ser.

Compromiso/implicación: La metáfora del jardinero con el significado dado por un futuro profesor de primaria de que el profesor guía y ayuda al crecimiento (Alarcón et al., 2014; Buaraphan, 2011; López-Luengo et al., 2015; Saban, 2010). Esta metáfora ha sido analizada en la literatura con un doble significado ya que puede significar ayuda al crecimiento en libertad, o también limitaciones al crecimiento por una poda estricta.

\subsubsection{Metáforas que reflejan emociones neutras}

Estas metáforas representan emociones como asombro y sorpresa. Un futuro profesor se identifica con el flautista de Hamelin y otro con una caja de sorpresa. Esta última metáfora es asignada a los niños por dos futuros maestros de infantil.

5.2.4. Metáforas que reflejan emociones primarias negativas

Destacamos que no han expresado ninguna metáfora de este tipo como miedo, ira, etc. 
LUCÍA MELLADO, MARÍA ROSA LUENGO, JUAN LUIS DE LA MONTAÑA Y MARÍA LUISA BERMEJO LAS METÁFORAS EMOCIONALES DE PROFESORES EN FORMACIÓN DE INFANTIL, PRIMARIA Y PSICOPEDAGOGÍA

5.2.5. Metáforas que reflejan emociones sociales negativas

Nerviosismo: La metáfora «tiemblo como un flan» expresa el nerviosismo que supone para un futuro profesor enfrentarse con sus primeras clases.

Desconcierto: Uno de los estudiantes expresa emociones de desconcierto y angustia al sentirse perdido como una cabra en un garaje, emociones típicas de los profesores en formación o principiantes (Cassel y Vincent, 2011; Russell y Hrycenko, 2006).

\section{CONCLUSIONES E IMPLiCACIONES}

La mayoría de los participantes han sido capaces de conceptualizar sus roles en forma de metáforas. Algunos de ellos han expresado metáforas correspondientes a más de una categoría, en algunos casos con significados muy distintos, especialmente en los maestros en formación. Esto puede deberse a que son estudiantes del primer curso del grado de maestro, que aún no han realizado sus prácticas de enseñanza y sus roles están menos definidos que los del último curso de psicopedagogía.

La mayor parte de las metáforas generales de los dos grupos de maestros se encuadran en la categoría de las conductistas/transmisivas, seguidas de las cognitivas/constructivistas y de las autorreferenciales. Estos dos grupos apenas expresan metáforas situadas.

Los resultados del grupo de psicopedagogía son muy diferentes de los dos grupos de maestro. Este grupo expresa el mismo número de metáforas conductistas/transmisivas que cognitivas/constructivistas, seguidas de las situadas y autorreferenciadas. Destacamos el gran aumento de las situadas respecto a las expresadas por los maestros.

La mayor parte de los estudiantes de psicopedagogía han realizado antes la diplomatura de maestro, en la que han realizado prácticas de enseñanza. Estos resultados nos indican la evolución de las metáforas en cuatro años de los futuros maestros, desde que entran en el primer curso universitario, hasta que realizan el último curso de psicopedagogía. Han evolucionado desde una enseñanza centrada en el profesor a otra más centrada en el alumno, haciendo más hincapié en el aprendizaje social que en el individual. Sin embargo, el trabajo de García et al. (2005) indica que apenas hay diferencias en la percepción de las emociones entre estudiantes de Maestro de $1 .^{\circ}$ y de $3 .^{\circ}$, por lo que son necesarios más estudios longitudinales para determinar esta evolución.

Otro resultado significativo, coincidente con anteriores estudios (Mellado et al., 2012), es que las metáforas no está asociadas a contenidos concretos, sino que son expresión de una visión general de la enseñanza y del rol del profesor, formada de sus propias experiencias como alumnos y de sus experiencias de enseñanza, en el caso del grupo de psicopedagogía. Este resultado coincide con 
LUCÍA MELLADO, MARÍA ROSA LUENGO, JUAN LUIS DE LA MONTAÑA Y MARÍA LUISA BERMEJO LAS METÁFORAS EMOCIONALES DE PROFESORES EN FORMACIÓN DE INFANTIL, PRIMARIA Y PSICOPEDAGOGÍA

el de Mahlios et al. (2010), que no encontraron diferencias significativas entre las metáforas de los profesores de primaria y secundaria.

En las metáforas emocionales el resultado es muy diferente que en las generales. En los tres grupos el mayor número de metáforas emocionales pertenecen a la categoría cognitiva/constructivista, especialmente en los maestros, seguida de la autorreferenciada, es decir, las que hacen referencia al yo. Los tres grupos expresan muy pocas metáforas emocionales en las categorías conductista-transmisiva y situada.

La mayoría de los participantes expresan en sus metáforas emociones positivas, como la emoción primaria de felicidad, aunque la mayoría expresan emociones positivas sociales como entusiasmo, diversión, seguridad, adaptación, estímulo, competencia, perseverancia, confianza, orgullo, comprensión, empatía, amor. Tres presentan emociones sociales neutras como la sorpresa, y muy pocos expresan emociones sociales negativas como el nerviosismo o el desconcierto. En la muestra analizada no hay emociones negativas primarias como el miedo o la ira. Estos resultados indican que los futuros profesores de nuestra muestra tienen una visión idealizada de la enseñanza (Hong, 2010) y aún no sienten las emociones negativas que llevan a algunos de sus colegas con mayor experiencia a sufrir el desgaste profesional.

Con respecto a las implicaciones, las metáforas se pueden convertir en una herramienta para que los futuros profesores reflexionen sobre sus concepciones, actitudes, emociones y modelos didácticos, especialmente durante las prácticas de enseñanza, pues las metáforas son un catalizador para la reflexión y pueden ayudar al profesorado a comprender y autorregular sus roles y sus acciones (Leavy et al., 2007; Russell y Hrycenko, 2006; Tannehill y MacPhail, 2014). Sin embargo, Mahlios et al. (2010) señalan la dificultad que tienen los profesores en formación inicial para cambiar sus metáforas, que están en gran parte determinadas por sus largos años de escolaridad como alumnos, por lo que sería necesario realizar programas de intervención para que los profesores en formación sean conscientes de sus propias metáforas, de su relación con sus concepciones, emociones y con su práctica en el aula. Esta intervención debería incidir en los procesos metacognitivos y metaemocionales, que promuevan las competencias emocionales de los futuros profesores (López et al., 2013; Repetto y Pérez-González, 2007) y que les doten de la capacidad de controlar y autorregular los cambios en sus propias metáforas y emociones (Seung et al., 2011; Sillman y Dana, 2001).

En cuanto a las implicaciones metodológicas, en futuros estudios sería conveniente ampliar los instrumentos de recogida y análisis de datos, incluyendo entrevistas, dibujos de las metáforas (Mellado et al., 2014), contraste entre las metáforas del profesor y la enseñanza, y las del alumno y el aprendizaje, así como la relación con otras variables como el género (Borrachero et al., 2014), que nos permitirían conocer la influencia que los estereotipos de género tienen en la profesión. 
LUCÍA MELLADO, MARÍA ROSA LUENGO, JUAN LUIS DE LA MONTAÑA Y MARÍA LUISA BERMEJO LAS METÁFORAS EMOCIONALES DE PROFESORES EN FORMACIÓN DE INFANTIL, PRIMARIA Y PSICOPEDAGOGÍA

Agradecimientos: Este trabajo ha sido financiado por el proyecto de investigación EDU2012-34140 del Ministerio de Economía y Competitividad del Gobierno de España y la Junta de Extremadura (España).

\section{REFERENCIAS BIBLIOGRÁFICAS}

Alarcón, P. y Díaz, C. (2014). El rol de la dinámica de fuerzas en la construcción reflexiva formal pasarse + dativo. Sintagma, 26, 7-21.

Alarcón, P.; Díaz, C.; Tagle, T.; Ramos, L. y Quintana, M. (2014). Metáforas para profesor y estudiante de pedagogía en un grupo de estudiantes de pedagogía chilenos. Revista Electrónica Actualidades Investigativas en Educación, 14 (2), 1-31.

Ben-Peretz, M.; Mendelson, N. y Kron, F. W. (2003). How teachers in different educational contexts view their roles. Teaching and Teacher Education, 19 (2), 277-290.

Bisquerra, R. (2000). Educación emocional y bienestar. Barcelona: Praxis-Wolters Kluwer.

Borrachero, A. B.; Brígido, M.; Mellado, L.; Costillo, E. y Mellado, V. (2014). Emotions in prospective secondary teachers when teaching science content, distinguishing by gender. Research in Science \& Technological Education, 32 (2), 182-215.

Boujaoude, S. (2000). Conceptions of science teaching revealed by metaphors and answers to open-ended questions. Journal of Science Teacher Education, 11 (2), 173-186.

Brígido, M.; Borrachero, A. B.; Bermejo, M. L. y Mellado, V. (2013). Prospective primary teachers' self-efficacy and emotions in science teaching. European Journal of Teacher Education, 36 (2), 200-217.

Buaraphan, K. (2011). Metaphorical roots of beliefs about teaching and learning science and their modifications in the standard based science teacher preparation programme. International Journal of Science Education, 33 (11), 1571-1595.

Bullough, R. V. Jr. (1992). Beginning teacher curriculum decisión making, personal teaching metaphors, and teacher education. Teaching and Teacher Education, 8 (3), 239-252.

Bullough, R. V. Jr. (2010). Parables, storytelling, and teacher education. Journal of Teacher Education, 61 (1-2), 153-160.

Cassel, D. y Vincent, D. (2011). Metaphors reveal preservice elementary teachers' views of mathematics and science teaching. School Science and Mathematics, 111 (7), 319-324.

Damasio, A. (2010). Y el cerebro creó al hombre. Barcelona: Editorial Destino.

De Bustos, E. (2000). La metáfora. Ensayos Transdisciplinares. Madrid: Fondo de Cultura Económica.

Díaz, J. L. y Flores, E. O. (2001). La estructura de la emoción humana: Un modelo cromático del sistema afectivo. Salud Mental, 24 (4), 20-35.

Duffé, A. L. (2004). Reflexiones psicolingüísticas y didácticas sobre el estudio de metáforas y modismos. Didáctica (Lengua y Literatura), 16, 33-44.

Fernández-Abascal, E.; Martín, M. y Domínguez, J. (2001). Procesos psicológicos. Madrid: Ediciones Pirámide.

Fuller, F. F. y Brown, O. H. (1975). Becoming a teacher. Enk. Ryan (Ed.). Teacher Education. Chicago: University of Chicago Press.

García, C. B.; Melero, M. P. T. y Salas, A. V. (2005). La Inteligencia Emocional en alumnos de Magisterio: La percepción y comprensión de los sentimientos y las emociones. Revista Interuniversitaria de Formación del Profesorado, 54, 169-194.

Goleman, D. (1996). Inteligencia emocional. Barcelona: Kairós. 
LUCÍA MELLADO, MARÍA ROSA LUENGO, JUAN LUIS DE LA MONTAÑA Y MARÍA LUISA BERMEJO LAS METÁFORAS EMOCIONALES DE PROFESORES EN FORMACIÓN DE INFANTIL, PRIMARIA Y PSICOPEDAGOGÍA

Gosselin, C. y Meixner, E. (2015). Blank Canvas and Glass Ceiling: Using Visual Metaphors and Narratives to Examine Preservice Teacher Development. Multicultural Perspectives, 17 (2), 73-80.

Gurney, B. F. (1995). Tugboats and tennis games: Preservice conceptions of teaching and learning revealed through metaphors. Journal of Research in Science Teaching, 32 (6), 569-583.

Gutiérrez, P. y Luengo, R. (2003). Orientación vocacional y género. Campo Abierto, 23 (1), $85-98$

Hong, J. Y. (2010). Pre-service and beginning teachers' professional identity and its relation to dropping out of the profession. Teaching and Teacher Education, 26 (8), 1530-1543.

Korthagen, F. A, (2010). La práctica, la teoría y la persona en la formación del profesorado. Revista Interuniversitaria de Formación del Profesorado, 68, 83-101.

Lakoff, G. y Johnson, M. (1986). Metáforas de la vida cotidiana. Madrid: Cátedra.

Lantolf, J. P. y Bobrova, L. (2014). Metaphor instruction in the L2 Spanish classroom: theoretical argument and pedagogical program. Journal of Spanish Language Teaching, 1 (1), 46-61.

Leavy, A. M.; McSorley, F. A. y Boté, L. A. (2007). An examination of what metaphor construction reveals about the evolution of preservice teachers' beliefs about teaching and learning. Teaching and Teacher Education, 23, 1217-1233.

López, A. M. M.; Barba, Á. V. y Díez, S. G. (2013). El registro de la emoción en el cuerpo, autoconocimiento y formación del profesorado. Revista Electrónica Interuniversitaria de Formación del Profesorado, 16 (2), 135-145.

López-Luengo, M. A.; Torrigo-Egido, L. M. y Vallés-Rapp, C. (2015). Qué metáforas personales definen al profesorado de Educación Infantil en formación. REICE. Revista Iberoamericana sobre Calidad, Eficacia y Cambio en Educación, 13 (3), 37-56.

Lorsbach, A. W.; Tobin, K.; Briscoe, C. y Lamaster, S. U. (1992). An interpretation of assesment methods in middle school science. International Journal of Science Education, 14 (3), 305-317.

Mahlios, M.; Massengill-Shaw, D. y Barry, A. (2010). Making sense of teaching through metaphors: a review across three studies. Teachers and Teaching: Theory and Practice, 16 (1), 49-71.

Marcos, F. (1993). Diccionario básico de recursos expresivos. Badajoz: Universitas Editorial.

Martínez, M. A.; Sauleda, N. y Huber, G. H. (2001). Metaphors as blueprints of thinking about teaching and learning. Teaching and Teacher Education, 17 (8), 965-977.

Mellado, L.; Bermejo, M. L.; Fajardo, M. I. y Borrachero, A. B. (2013). Las metáforas emocionales de los estudiantes de educación primaria y educación infantil. International Journal of Development and Educational Psychology, 1 (1), 281-289.

Mellado, L.; Bermejo, M. L. y Mellado, V. (2012). Personal metaphors of prospective secondary economics and science teachers. Asia Pacific Journal of Teacher Education, 40 (4), 395-408.

Mellado, L.; Luengo, M. R.; De la Montaña, J. L.; Borrachero, A. B. y Bermejo, M. L. (2014). Las metáforas emocionales de estudiantes de Psicopedagogía sobre el profesor y el aprendizaje. Campo Abierto, 33 (1), 153-170.

Rebollo, M. A.; García, R.; Barragán, R., Buzón, O. y Vega, L. (2008). Las emociones en el aprendizaje online. Relieve, 14 (1).

Rebollo, M. A.; Jiménez, R.; Sabuco, A. y Vega, L. (2013). Metáforas que usan las profesoras en los procesos de cambio educativo con perspectiva de género. Profesorado: Revista de Currículum y Formación del Profesorado, 17 (1), 43-56. 
LUCÍA MELLADO, MARÍA ROSA LUENGO, JUAN LUIS DE LA MONTAÑA Y MARÍA LUISA BERMEJO LAS METÁFORAS EMOCIONALES DE PROFESORES EN FORMACIÓN DE INFANTIL, PRIMARIA Y PSICOPEDAGOGÍA

Repetto, E. R. y Pérez-González, J. C. (2007). Formación en competencias socioemocionales a través de las prácticas en empresas. Revista Europea de Formación Profesional, 40, $92-112$

Ritchie, S. M. (1994). Metaphor as a tool for constructivist science teaching. International Journal of Science Education, 16 (3), 293-303.

Ritchie, S. M.; Aubusson, P. J. y Harrison, A. G. (2006). Metaphorically thinking, En P. J. Aubusson, A. G. Harrison y S. T. Ritchie (Eds.). Metaphor and analogy in science education (pp. 189-195). Dordrecht: Springer.

Russell, T. y Hrycenko, M. (2006). The role of Metaphor in a new science teacher's learning from experience. En P. J. Aubusson, A. G. Harrison y S. T. Ritchie (Eds.). Metaphor and analogy in science education (pp. 131-142). Dordrecht: Springer.

Saban, A. (2010). Prospective teachers' metaphorical conceptualizations of learner. Teaching and Teacher Education, 26 (2), 290-305.

Seung, E.; Park, S. y Narayan, R. (2011). Exploring elementary pre-service teachers' beliefs about science teaching and learning as revealed in their metaphor writing. Journal of Science Education and Technology, 20 (6), 703-714.

Shaw, D. M.; Barry, A. y Mahlios, M. (2008). Preservice teachers' metaphors of teaching in relation to literacy beliefs. Teachers and Teaching. Theory and Practice, 14 (1), 35-50.

Sillmam, K. y Dana, T. M. (2001). Metaphor: a tool for promoting prospective elementary teachers' participation in science teacher learning community. Journal of Science Teacher Education, 12 (2), 87-106.

Tannehill, D. y MacPhail, A. (2014). What examining teaching metaphors tells us about preservice teachers' developing beliefs about teaching and learning. Physical Education and Sport Pedagogy, 19 (2), 149-163.

Thomas, L. C. y Beauchamp, C. (2011). Understanding new teachers' professional identities through metaphor. Teaching and Teacher Education, 27 (4), 762-769.

Tobin, K. (2012). Sociocultural perspectives on science education. En B. J. Fraser, K. Tobin y C. J. McRobbie (Eds.). Second International Handbook of Science Education (pp. 3-17). Dordrecht: Springer.

Tobin, K. y Tippins, D. J. (1996). Metaphors as seeds for conceptual change and the improvement of science education. Science Education, 80 (6), 711-730.

Tobin, K.; Tippins, D. J. y Gallard, A. J. (1994). Research on instructional strategies for teaching science. En D. Gabel (Ed.). Handbook of Research on Science Teaching and Learning (pp. 3-44). New York: MacMillan.

Treagust, D. F. y Harrison, A. G. (2000). In search of explanatory frameworks: an analysis of Richard Feynman's lecture "Atoms in motions". International Journal of Science Education, 22 (11), 1157-1170.

Zembylas, M. (2004). Emotion metaphors and emotional labor in science teaching. Science Education, 88 (3), 301-324.

Zhao, H.; Coombs, S. y Zhou, X. (2011). Developing professional knowledge about teachers through metaphor research: facilitating a process of change. Teacher Development, 14 (3), 381-395. 
